\title{
Valuation of sitting and standing in metro trains using revealed preferences
}

\begin{abstract}
The estimation of differences in the value of in-vehicle time sitting and standing is usually made with stated choice (SC) data, partly due to the lack of revealed preference data. In this paper, we use the observed behaviour of a subset of metro users in Singapore, who are willing to travel a longer time (into the opposite direction or backwards) to secure a seat for the actual trip in the direction towards their destination. We use smart card transactions to estimate the share of users who are willing to travel in the opposite direction during the first part of their trip and the average train occupancy per section to estimate differences in the valuation of travel time sitting and standing - translated into a standing multiplier or standing premium, which is analogous to the crowding multiplier that is usually found in the crowding valuation literature. We find that the standing multiplier is between 1.18 and 1.24 with the current crowding levels in the morning peak and can be as much as 1.55 with a density of 3 standing passengers per square metre. The results are compared to previous SC studies from other countries. The values found here are an indication of a standing premium that can be used to assess the social benefit of increasing the seat capacity of a public transport system and of applying peak spreading strategies.
\end{abstract}

Keywords: revealed preference, sitting, standing, crowding, public transport, metro 


\section{Introduction}

Mode and route choice decisions in transport have traditionally been modelled and evaluated according to cost and various elements related to travel time. Going beyond monetary cost and time, additional factors associated with the riding comfort and service reliability of public transport systems have shown to be relevant for mode, route and activity scheduling choices in various studies (e.g., Bates et al., 2001; Kim et al., 2009; Li and Hensher, 2011; Raveau et al., 2011; Wardman and Whelan, 2011; Börjesson et al., 2012; Theler and Axhausen, 2013; Tirachini et al., 2013; Batarce et al., 2015). Among the factors capturing riding comfort, seat availability and perceptions of crowding levels are regarded to have significant behavioural impacts. As such factors are decisive mostly in urban contexts, for which continuous growth is expected globally (United Nations Department of Economic and Social Affairs 2010), it is fair to assume that those behavioural aspects will become even more relevant for transport policy in the future. Additionally, increasing income levels in both developing and developed countries also suggest that more weight will be assigned to the quality and comfort features of public transport trips.

For users, standing is usually less comfortable than sitting, especially for long trips; therefore, we would expect users to be willing to pay more to reduce travel time when standing rather than sitting. Such an outcome has been shown in the literature on users' valuation of sitting, standing and crowding ${ }^{1}$. Numerous studies, such as Douglas and Karpouzis (2005), Whelan and Crockett (2009), Kim et al. (2009), Hensher et al. (2011), Fröhlich et al. (2012), Tirachini et al. (2013) and Batarce et al. (2015), show the crowding disutility that arises when the occupancy levels of vehicles and stations increase over a particular threshold. The most common procedure to estimate this crowding disutility is the use of discrete choice models with for stated choice (SC) data.

In this paper, we estimate the differences in the valuation of sitting and standing during public transport trips using revealed preferences (RP) of a subset of metro users in Singapore, who are willing to take a train in the opposite direction of their destination (backwards) to secure a seat during their travel towards their destination (forwards) after the train changes directions at the terminal stop of the line. This is a novel choice situation for analysing standing and crowding disutilities based on observed behaviours. In other words, some passengers are willing to spend more time in transit for a more comfortable ride, which differs from previous RP studies that analysed the substitution between waiting time and in-vehicle time when studying crowding externalities (LT Marketing, 1998; Kroes et al., 2014). In particular, we use these RP data to

\footnotetext{
${ }^{1}$ In general, some passengers might prefer to travel standing rather than sitting, particularly for short trips; nonetheless, the literature shows that, on average, there is a travel time disutility associated with crowding and standing.
} 
estimate the impact of crowding and the valuation of seat availability for mass rapid transit (MRT) route choice decisions, and we find a standing multiplier or standing premium that depend on the load factor and density of standees per time period and section - similar to the crowding multiplier usually found in the crowding valuation literature based on SP experiments. Multinomial logit ( $\mathrm{MNL}$ ) route choice models are estimated based on peak hours observations, where a share of commuters prefer to travel a short distance in the opposite direction to ensure a more comfortable, seated ride to the final destination. Individual choices are not modelled; instead, they are used to estimate the share of passengers who decide to travel backwards depending on the origin, destination and length of the trip.

Second, we present a methodology to infer the routes actually taken and the vehicle loads expected in situations in which smart card transaction cannot be directly traced back to the individual vehicles and are only observed at the level of public transport stops, as is usually the case of MRT systems. This methodology allows us to take advantage of the smart card transactions database, which contains all the records of entries (tap-ins) and exits (tap-outs) to and from stations in Singapore's MRT system. Reliable high-resolution RP data are very valuable as a way to obtain the economic values of service quality attributes, such as the value of avoiding crowded travel time, as it is based on estimations of actual behaviours rather than reported behaviours.

The remainder of the article is organized as follows. Section 2 presents a literature review on crowding and standing valuations. In Section 3, we describe our smart card dataset and the methodology to estimate travel times, trainloads and the proportion of passengers who initially travel backwards (away from their final destination to secure a more comfortable ride). Section 4 presents and discusses the choice models that are used for the estimation of the disutilities of time sitting and time standing. The results are compared with previous outcomes from studies in the United Kingdom and France. Regarding policy implications, Section 5 analyses the influence of observed standing and crowding externalities on supply levels of public transport services. Finally, Section 6 provides a summary and the main conclusions of the study.

\section{Literature review}

A common objective of crowding valuation studies is the estimation of a crowding multiplier, that is, the ratio between travel time parameters under crowded and uncrowded conditions. In crowding valuation studies, the standard procedure is to define a crowding attribute that interacts with travel time in linear or non-linear functional forms to capture the effect of increased crowding discomfort during longer trips. As the crowding phenomenon relates to station and vehicle occupancy, constructs that assess occupancy levels are used, such as the load factor (i.e., the total number of passengers over the number of seats) and the density of 
standees per square metre ${ }^{2}$. An example of utility function that can be used to assess the crowding discomfort in public transport vehicles is the following:

$$
U=\alpha_{0}+\beta_{1} \cdot t+\beta_{2} \cdot t \cdot S+\beta_{3} \cdot t \cdot C r+\beta_{4} \cdot t \cdot C r \cdot S+\varepsilon
$$

where $\alpha_{0}$ is an alternative specific constant; $t$ is in-vehicle time; $S$ is a dummy variable that equals 1 if the passenger has to stand and 0 if the passenger is able to sit; $C r$ is a variable that describes the occupancy level of passengers; $\beta_{i}$ are the passenger taste parameters; and $\varepsilon$ is a random error. If choice follows a multinomial logit (MNL) model, with expression (1), the crowding multiplier is defined as follows:

$$
C M=\frac{\beta_{1}+\beta_{2} \cdot S+\beta_{3} \cdot C r+\beta_{4} \cdot C r \cdot S}{\beta_{1}}
$$

The crowding multiplier increases in value as crowding worsens. Some studies aim to estimate average crowding multipliers that increase with occupancy levels, regardless of whether the concerned passenger is sitting or standing (e.g., Hensher et al., 2011; Basu and Hunt, 2012; Tirachini et al., 2013). Other studies - such as Douglas and Karpouzis (2006) on trains in Sydney, Australia, Kroes et al. (2014) on trains and buses in the Paris region, and several papers and reports on the rail industry in Britain (for a review and meta-analysis, see Wardman and Whelan, 2011) - estimate different in-vehicle time parameters for passengers who are sitting and standing, as a function of a measure of vehicle occupancy levels. For example, Whelan and Crockett (2009) estimated crowding multipliers for rail services in Great Britain, focusing on London and the Southeast; for seated passengers, the crowding multiplier increases from 1.0 to 1.54 as the density of standing passengers increases from 0 to 6 passengers per square metre $\left(\mathrm{pax} / \mathrm{m}^{2}\right)$, whereas the crowding multiplier is between 1.43 and 2.21 for standing passengers. Therefore, standing passengers in uncrowded conditions value travel time savings 43 per cent more than seated passengers ${ }^{3}$. Lower crowding multipliers have recently been found by Kroes

\footnotetext{
2 The load factor is more commonly used due to the easiness of its computation. However, it does not provide a clear indication of the degree of crowding suffered by passengers, which can be more accurately captured by estimating the density of standees per square metre. For example, a load factor of 200 per cent, relative to the seating capacity, indicates that one of two passengers is standing, but it is not clear how uncomfortable the situation is for those standing. However, a standing density of five passengers per square metre is a very likely indicator of crowding discomfort, regardless of the size of the vehicle or the number of seats. On the other hand, crowding disutility may also be present before all seats are occupied; see Wardman and Whelan (2011) and Tirachini et al. (2013).

${ }^{3}$ In busy metro systems in Latin America and Asia, the passenger density inside trains can reach beyond 6 pax $/ \mathrm{m}^{2}$. Basu and Hunt (2012) provide images of 4, 7 and 12 men standing inside a square metre, which were used in a stated preference study to estimate the value of time savings on increasing crowding conditions in Mumbai, India. In reality, maximum passenger densities in public transport services are constrained by the fact that some
} 
et al. (2014) in the Paris region (Île-de-France), with maximum values of 1.4 for sitting and 1.6 for standing combining data from all public transport modes (metro, train and bus).

Beyond these articles and reports, crowding penalties have also been included by a handful of countries in their official guidelines for transport project assessment, as reviewed by OECD/ITF (2014). For example, in Australia, the crowding multiplier is up to 1.3 for sitting and up to 2.0 for standing at maximum occupancy. In France, crowding multipliers increase linearly as a function of the passenger density per square metre, with values of 1.3 for sitting and 1.6 for standing with 4 passengers per square metre $\left(\mathrm{pax} / \mathrm{m}^{2}\right)$. In Sweden, the crowding multiplier is up to 3.0, whilst in the United Kingdom it is up to 2.1 for sitting and 2.8 for standing, with 3 $\operatorname{pax} / \mathrm{m}^{2}$.

In the existing literature, the vast majority of evidence regarding how crowding influences mode and route choice decisions is based on stated choice (SC) experiments, which are performed to estimate discrete choice models. Reliable preference data on people's choices of the mode and time of day for travelling, as a function of the occupancy levels of public transport alternatives, are difficult to obtain. Modelling the behavioural impact of crowding based on revealed preference data requires both knowledge of the dynamic state of the transport system and users' decisions. Alternatively, if count data are unavailable, additional variables that describe the equilibrium behaviour endogenously can be included in choice modelling, as demonstrated by Timmins and Murdock (2007) regarding location choice problems; however, the application of their model in the context of route choice would require some adjustments because of the additional complexity of overlapping route alternatives. While the first data requirement can be fulfilled with automated passenger count systems, such systems are very rarely installed in all vehicles that provide full coverage of a study region. At the same time, the observed public transport trips will need to be described in adequate detail to identify the individual vehicle that actually has been taken. For conventional travel surveys, such detail is usually not possible, given the limitations of the respondents reporting trips' start times and exact routes; however, more innovative approaches employing GPS tracing could improve the situation. However, with the advent and pervasive use of smart cards for fare collection in public transport systems, both dynamic vehicle loads and route choice decisions can be obtained from the same data source.

In spite of data limitations, we found three previous studies that have reported the use of revealed choices to analyse the influence of crowding on travel decisions. LT Marketing (1988), as cited by Wardman and Whelan (2011), observed situations in which passengers did not board a crowded train and waited for a less crowded or empty train in the London

passengers carry bags, suitcases, rucksacks, etc., which increase the projected floor area that a passenger occupies (TRB, 2003). 
Underground; they reported standing multipliers between 1.4 and 2.7, and seated multipliers between 1.0 and 1.6, i.e., values that are not significantly different from the other 16 stated preference British studies summarized by Wardman and Whelan (2011). Kroes et al. (2014) also analyse the number of passengers who wait for a second train to avoid boarding a crowded train in Paris. Although Kroes et al. (2014) do not estimate a RP choice model, they do observe that the actual shares of passengers who are willing to wait for the next train to avoid crowded conditions are lower than the shares implied by the SP values, which points to a possible overestimation of the SP crowding disutility values in this context. Finally, a model reported by Batarce et al. (2015) combines stated preferences with revealed preference data (from route choice observations) in Santiago, Chile, and finds a crowding multiplier of approximately 2.0 when the passenger density is between 5 and $6 \mathrm{pax} / \mathrm{m}^{2}$ in buses and metro trains, regardless of whether the traveller is sitting or standing.

\section{Data description}

Individual data from smart card transactions are described and analysed in Sections 3.1 and 3.2, which are used to estimate the shares of passengers travelling backwards and forwards per origin-destination pair (Section 3.3) and the train loads per section (Section 3.4), to estimate MNL models (Section 4), from which one can obtain standing multipliers that are sensitive to the occupancy levels of trains.

\subsection{Travelling backwards and forwards}

Because of the high spatial-temporal resolution, smart card data have been widely used to study the travel behaviour and patterns of individual users (e.g., Chakirov and Erath, 2011; Pelletier et al., 2011; Hasan et al., 2012; Munizaga and Palma, 2012; Sun et al., 2013). In this study, we use a smart card record of all MRT journeys in Singapore, collected over one week (from 11 April 2011 to 17 April 2011), for a total of 1,994,714 distinct MRT users. As train passengers have to tap the same smart card each time they enter or leave any MRT station, the dataset contains records of the time and location for every single trip's tap-in and tap-out. Previously, Chakirov and Erath (2011) provided a detailed analysis of the same dataset, focusing on characterizing the public transport system and travel behaviours in Singapore. Chakirov and Erath (2011) depicted the distribution of passengers' waiting times by identifying the passenger with the shortest travel time as a reference point and assuming that he/she boarded a train immediately without waiting on the platform (zero waiting time). Therefore, the difference in travel time between other passengers and the reference passenger is assumed to be waiting time. One of the interesting findings of Chakirov and Erath (2011) was a double-peaked distribution of waiting times at certain MRT stations. These MRT stations are surrounded by high-density residential estates with more than 100,000 people living in the catchment area of the MRT stop and are the second or third stop at the beginning or end of an MRT line. The 
'waiting times' were extracted by Chakirov and Erath (2011) using the shortest recorded travel time between two stations of the same line as a benchmark. This double-peaked distribution can be prominently observed at the second station (STN2) of eastbound services of the islandcrossing East-West line ${ }^{4}$, as depicted in Figure .

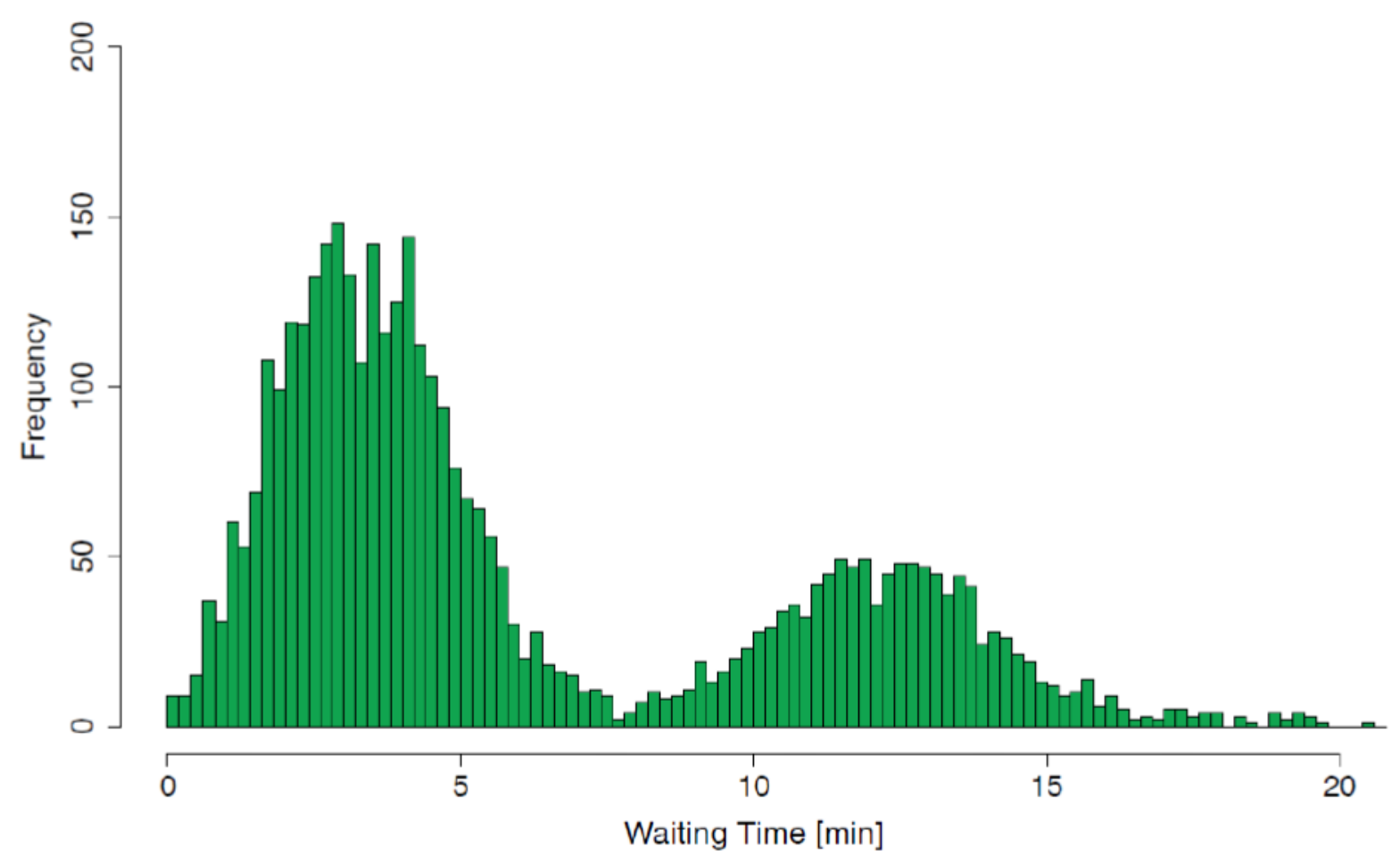

Figure 1: Histogram of waiting times at STN2 for trips between 7 am and 9 am; source: Chakirov and Erath (2011)

Due to the high density residential development around the MRT stations, trains tend to fill up quickly with commuters heading towards the city centre, which features a high job density, during the morning peak hour. As introduced in Chakirov and Erath (2011), the double-peaked shape of the 'waiting time' distribution, as shown in Figure 1, results from the following two choices:

1) Travelling directly (forwards) to one's destination, which corresponds with the larger peak between 1 and 6 minutes in Figure 1.

2) Initially travelling in opposite direction of one's desired destination (backwards) to secure a seat at the first station (STN1) of the line and then travelling forwards to one's final destination. This behaviour is detected as the second peak in the waiting time

\footnotetext{
${ }^{4}$ For more information on the stations of the East-West MRT line, please see Chakirov and Erath (2011) and the network map at www.mrt.cl
} 
distribution, approximately 10 to 15 minutes in Figure 1 . A simple representation of this behaviour is shown in Figure 2.

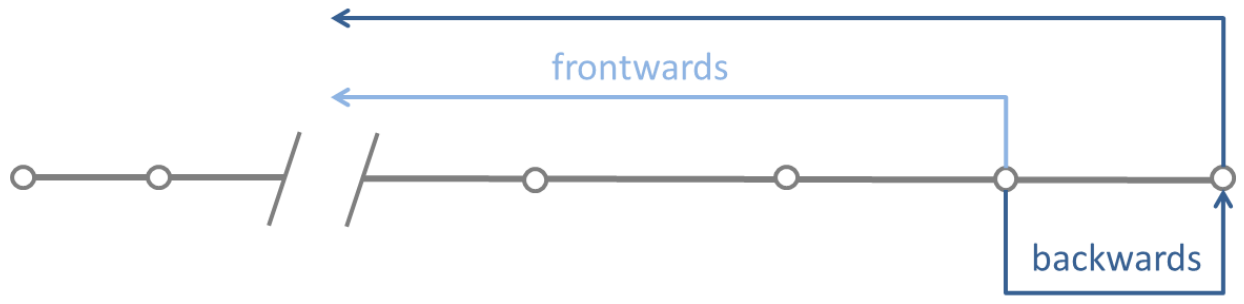

Station $4 \quad$ Station $3 \quad$ Station $2 \quad$ Station 1

\section{Figure 2: Illustration of the studied metro service and the 'travelling backwards' phenomenon}

Therefore, the observed 'waiting time' consists of two parts: (1) real waiting time on the platform for passengers travelling forwards and (2) waiting time on the opposite platform plus additional travelling costs for passengers travelling backwards. As a result, the distribution shown in Error! Reference source not found. is a consequence of choices (1) and (2) combined. The difference of the mean between distributions (1) and (2) is due to the additional travel time from STN2 (Station 2) to STN1 (the terminal station), slack/recovery time at STN1 and the return travel time to STN2 on the eastbound service. The variance of distribution (1) depends on the variation in the frequency of westbound service and the variation in the travel times between stations. The variance of distribution (2) depends, similar to distribution (1), on the service frequency of eastbound service, the travel times between stations and the operational variations in slack/recovery time at the terminal station (STN1). Therefore, the variance of distribution (2) can be expected to be slightly higher, as Figure 1 shows.

The same behaviour can also be observed among passengers who board at a few other stations, though such behaviour is less pronounced. Furthermore, it is worth mentioning that the station design and direction change procedure favours such behaviour, as passengers are not required to exit the train at the terminal station. Passenger surveys would have helped confirm this dual behaviour among users; however, in this study, we must rely on smartcard data, as we do not count with surveys. However, all these findings can be easily observed by travelling on the Singapore's eastbound MRT service between STN2 and STN1 on any given regular workday during the morning rush hour. To understand users' preferences regarding these two choices, we model travel times to analyse an individual's choice from his/her smart card transactions to obtain aggregated shares of the passengers who travel backwards and forwards. 


\subsection{Modelling metro travel time}

Essentially, in smart card transactions, a metro journey starts with a tap-in at a fare gate in the origin station. Once a user finishes his/her journey by tapping-out with the same smart card, a record with full spatial-temporal resolution is created, registering the detailed time and location information of the corresponding journey. Because Singapore employs a distance-based fare scheme for public transport systems, the actual travel distance for each stage is also registered. Table 1 presents the fields and their contents of the smart card data used in this study. To simplify the modelling estimations, we only consider trips that have origin and destination stations in the EW line, i.e., the subway route choice is not modelled.

Table 1. Fields and descriptions of smart card data

\begin{tabular}{|l|l|}
\hline Field & Description \\
\hline Trip ID & A unique number for each MRT trip \\
\hline Card ID & A unique number for each smart card (anonymous) \\
\hline Passenger Type & The attribute of cardholder (Adult, Senior Citizen and Child) \\
\hline Boarding Station & Boarding station \\
\hline Alighting Station & Alighting station \\
\hline Ride Date & Trip date (e.g., '2011-04-11') \\
\hline Ride Start Time & Tap-in time (e.g., '08:00:00') \\
\hline Ride End Time & Tap-out time (e.g., '08:00:00') \\
\hline Ride Distance & Trip distance (e.g., '12.0 km') \\
\hline
\end{tabular}

The comprehensive trip records allow us to reconstruct each metro trip in its spatial and temporal dimensions. To quantify the metro service level and to understand the demand patterns of the EW service, Sun et al. (2012) proposed a regression model to estimate the spatial-temporal density of metro users based on a simplified travel time model, with the train speed $v$, the dwell time $D w$ (including acceleration and deceleration) and the average access (egress) time as parameters. In this paper, we adapt this model to better estimate the desired (shortest) travel time. Essentially, a metro trip is divided into four stages, as shown in Figure 1. 


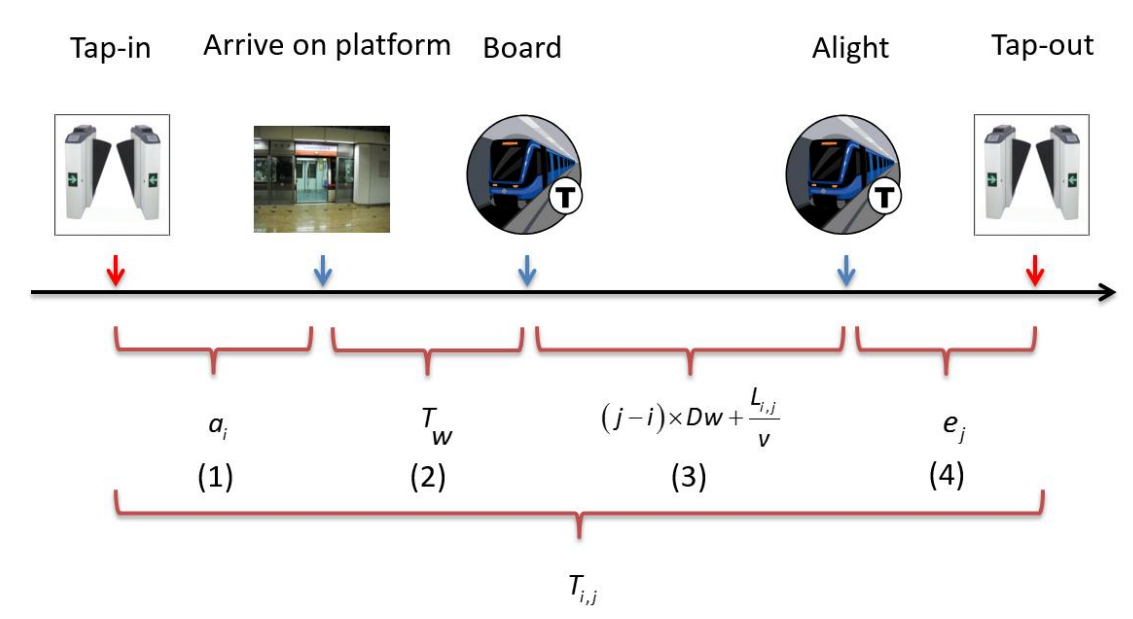

Figure 1: Modelling travel time of metro trips

Let $T_{i j}$ be the total journey time from station $i$ to station $j$ (the inter-event time between tapping-in at $i$ and tapping-out at $j$ ), which is divided in the following components:

(1) access time $a_{i}$ from fare gate to platform;

(2) waiting time at platform $T_{w}$;

(3) in-vehicle time $t^{i j}=\sum_{k=i}^{j-1} t^{k, k+1}=\sum_{k=i}^{j-1}\left(D w+\frac{L_{k, k+1}}{v}\right)$, where $L_{k, k+1}$ is the distance between two consecutive stations $k$ and $k+1, D w$ is the average dwell time at each station and $v$ is the train's cruising speed; and

(4) egress time $e_{j}$ from platform to fare gate at destination station $j$.

Taken together, the total journey time can be formulated as follows:

$$
T_{i j}=a_{i}+T_{w}+\left((j-i) \times D w+\frac{L_{i, j}}{v}\right)+e_{j}
$$

where $L_{i, j}=\sum_{k=i}^{j-1} L_{k, k+1}$.

The parameters in Equation (3) have to be estimated using our smart card database. We apply the same strategy to regress the model, as in Sun et al. (2012), by selecting the top-10 fastest passengers with shortest travel times for each origin-destination (OD) pair as sample observations for the regression and assuming that $T_{w}=0$ for these users. On the other hand, 
the time cost for access $a_{i}$ and egress $e_{i}$ are comparable to each other for stations with one entrance (exit). However, if a station is equipped with multiple entrances (exits), the time cost for access and egress might be different because of the variation in walking time to different gates. For the sake of simplicity, we assume that the access time and egress time are equal for any station $i$ :

$$
a_{i}=e_{i}, \quad \forall i
$$

Therefore, we apply a general linear regression to obtain the estimated values for the two universal parameters $D w=71.19 \pm 0.80 s$ (estimation at a $95 \%$ confidence interval $(\mathrm{Cl})$, with $R^{2}=0.9997 ; \hat{v}=22.19 \mathrm{~m} / \mathrm{s}$ with a $95 \% \mathrm{Cl}$ as $\left.[21.88,22.51]\right)$. Note that the reported operation speed of metro trains in Singapore is approximately $85 \mathrm{~km} / \mathrm{h} \approx 23.6 \mathrm{~m} / \mathrm{s}$, which suggests that the new model provides good estimations. In fact, more variation, such as longer dwell times, could result from excessive crowding during rush hours (Lin and Wilson, 1992), such that trains are not always on time. In Singapore's case, metro services are automatically or semiautomatically operated, making them more resilient to disturbances by adjusting the speed and dwell time in real-time. Thus, we still use this model to analyse passenger travel time. The estimated access (egress) times for each station are shown in Figure 2.

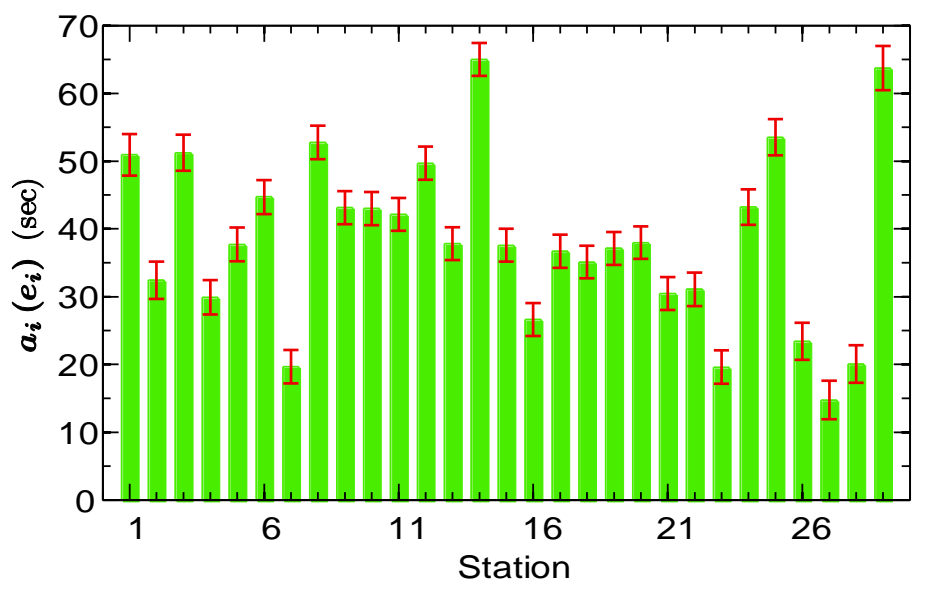

Figure 2. Access and egress time for each station along the East-West line.

\subsection{Estimating the share of passengers travelling backwards}

Passengers are divided in two groups:

- G1: those who travel forwards to their destination.

- G2: those who travel backwards to the first station, wait and then travel forwards to their destination. 
Using the passengers with the shortest travel times as reference points (with $T_{w} \approx 0$ ), we estimated the waiting time distributions $P_{i j}\left(T_{w}\right)$ for all the users travelling on the OD pair $(i, j)$ . Note that, for users travelling backwards (G2), $T_{w}$ is no longer the waiting time; $T_{w}$ is the total extra cost, including all the time involved in travelling backwards (i.e., waiting time for the train going backwards, in-vehicle time in this train, waiting time at STN1, and in-vehicle time travelling forwards from STN1 to the final destination).

To illustrate the influence of trip length on users' behaviours regarding travelling forwards (G1) or backwards and then forwards (G2), Figure 3 shows the waiting time distribution at STN2, STN3 and STN4 obtained from the smart card data (with destinations at STN5, STN7, STN15 and STN17). A significant double-peaked waiting time distribution is found for long travel distances for users departing from STN2 and STN3, which points to an increase in the share of passengers travelling backwards as the (standing) trip distance increases. The second peak vanishes for users who depart from STN4 (Figure 5c), which indicates the existence of a distance threshold related to the decision of travelling backwards: from STN4 onwards, the number of users who travel backwards is negligible or zero. 

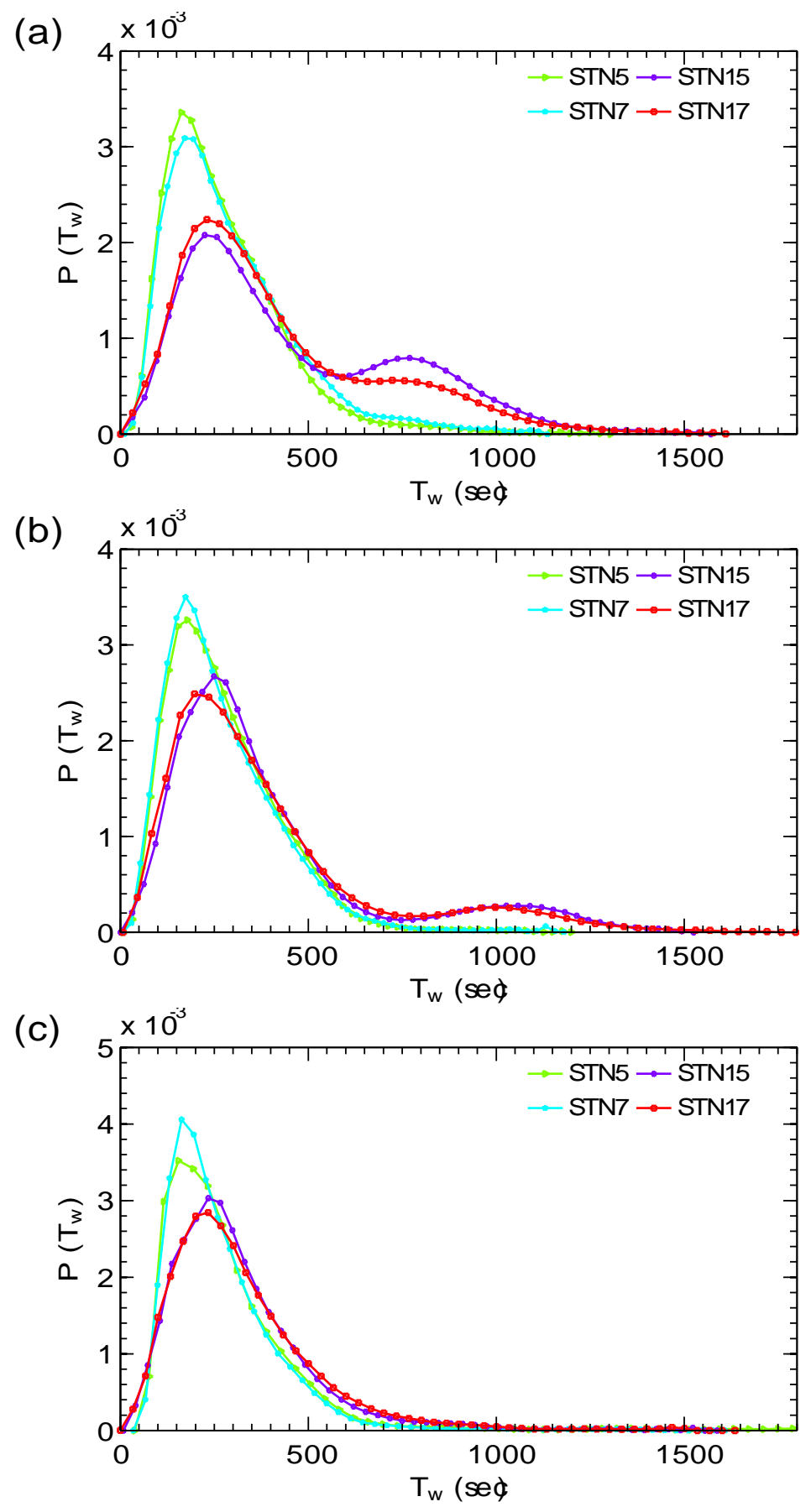

Figure 3. Waiting time distribution from STN2 (top panel), STN3 (middle panel) and STN4 (bottom panel) to different destinations

Given the behaviour revealed in Figure 5, the next problem involves estimating the proportion of passengers who choose each alternative (travelling forwards versus travelling backwards and then forwards) for a given OD pair. In other words, we need to estimate the parameters for a 
mix of two distributions with the proportions of passengers given by $\alpha_{F}$ (G1, the proportion of passengers travelling forwards) and $\alpha_{B}(\mathrm{G} 2$, the proportion of passengers travelling backwards), such that $\alpha_{F}+\alpha_{B}=1$ :

$$
f_{i j}\left(T_{w}\right)=\alpha_{F} f_{i j}^{F}\left(T_{w}\right)+\alpha_{B} f_{i j}^{B}\left(T_{w}\right)
$$

where $f_{i j}\left(T_{w}\right)$ is the observed waiting time distribution (as in Figure 3), $f_{i j}^{F}\left(T_{w}\right)$ characterizes the waiting time distribution of passengers travelling forwards and $f_{i j}^{B}\left(T_{w}\right)$ presents the probability density function of the 'waiting time' when passengers travel backwards first. For the purpose of choice modelling, we are interested in only the proportion parameters $\alpha_{F}$ and $\alpha_{B}$. However, in this case, the 'waiting time' distributions $f_{i j}^{F}\left(T_{w}\right)$ and $f_{i j}^{B}\left(T_{w}\right)$ are also known to us. If the distributions $f_{i j}^{F}\left(T_{w}\right)$ and $f_{i j}^{B}\left(T_{w}\right)$ can be characterized by deterministic forms (such as normal or log-normal), the estimation can be considered a mixture distribution problem that can be solved by applying the expectation maximization (EM) algorithm (Dempster et al., 1977). However, in practice, it is difficult to determine a universal probability density function form with a limited number of parameters to characterize component distributions $f_{1}\left(T_{w}\right)$ and $f_{2}\left(T_{w}\right)$ for all OD pairs, even though we can sketch the shape of $f_{i j}\left(T_{w}\right)$ from smart card data. Therefore, we do not directly apply a conventional likelihoodbased parametric estimation approach to estimate the proportions. Alternatively, by taking a closer look at the travel time composition of the forwards and backwards and then forwards alternatives, we note that the travel time difference (additional cost $\Delta T_{i}$ at station $i$ ) is characterized by the following:

$$
\Delta T_{i}=2 t^{1 i}+t_{h}
$$

where $t_{h}\left(t_{h}>0\right)$ is the train slack or recovery time at terminal STN1. Therefore, we have the following approximated probability density function:

$$
\begin{aligned}
& f_{i j}\left(T_{w}\right)=\left(1-\alpha_{B}\right) f_{i j}^{F}\left(T_{w}\right)+\alpha_{B} f_{i j}^{F}\left(T_{w}-\Delta T_{i}\right) \\
& =\left(1-\alpha_{B}\right) f_{1 j}^{F}\left(T_{w}\right)+\alpha_{B} f_{1 j}^{F}\left(T_{w}-\Delta T_{i}\right),
\end{aligned}
$$

where $f_{1 j}^{F}\left(T_{w}\right)$ is probability density function (p.d.f.) of the waiting time of passengers travelling from terminal STN1 to station $j$. Additionally, the cumulative distribution function (c.d.f.) can be estimated as a function of $\alpha_{B}$ :

$$
F_{i j}\left(T_{w} ; \alpha_{B}\right)=\left(1-\alpha_{B}\right) F_{1 j}^{F}\left(T_{w}\right)+\alpha_{B} F_{1 j}^{F}\left(T_{w}-\Delta T_{i}\right),
$$


where $F_{1 j}^{F}\left(T_{w}\right)$ is the empirical cumulative distribution function of the waiting time. In doing so, we can replace the unknown waiting time distribution with its empirical c.d.f obtained from the smart card transactions. Therefore, the estimation problem takes the waiting time distributions as known facts and only the proportions as parameters to be estimated. To obtain the proportion parameter $\alpha_{B}$, we define the best estimation as $\hat{\alpha}_{B}$, which minimizes the Kolmogorov-Smirnov (KS) statistic:

$$
\hat{\alpha}_{B}=\underset{\alpha_{B}}{\arg \min }\left(\sup _{t}\left|F_{i j}\left(t ; \alpha_{B}\right)-G_{i j}(t)\right|\right)
$$

where $G_{i j}(t)$ is the empirical c.d.f of the waiting time from station $i$ to station $j$. Given any $\alpha_{B}$, we can obtain the corresponding KS value as that in Equation (9). Therefore, in a predefined range (for example, from 0 to 1 , with an interval of 0.001), we can calculate the KS values for all $\alpha_{B}$, and the best estimation $\hat{\alpha}_{B}$ can be determined by finding the $\alpha_{B}$ with the minimum KS value. In Figure 4 , we show the observed c.d.f and p.d.f of $T_{w}$ from STN2 to STN14, against the corresponding estimation by using the proposed inferential model. Figure $6 \mathrm{~b}$ shows that $28.9 \%$ of passengers are predicted to travel backwards from STN2 to STN1 when the final destination is STN14.

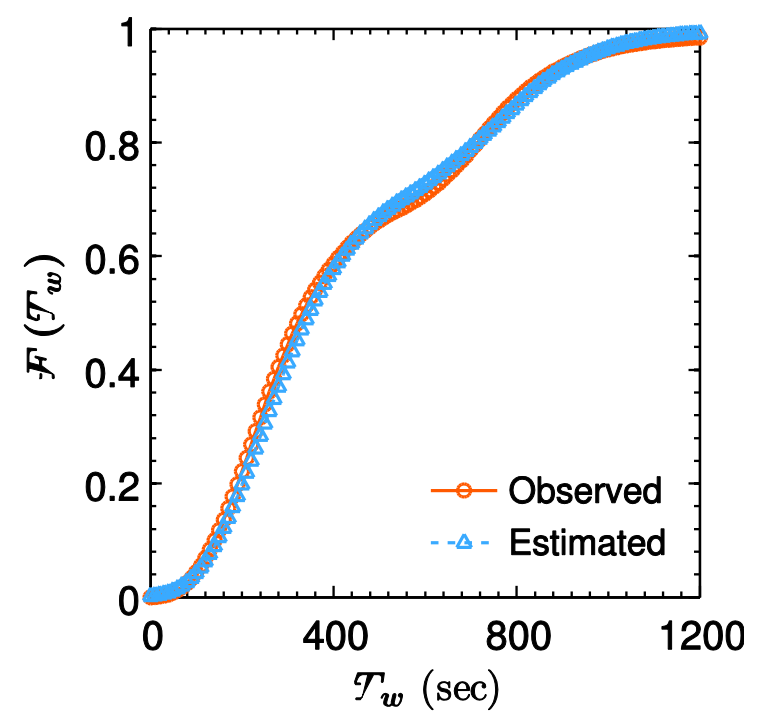

(a) c.d.f

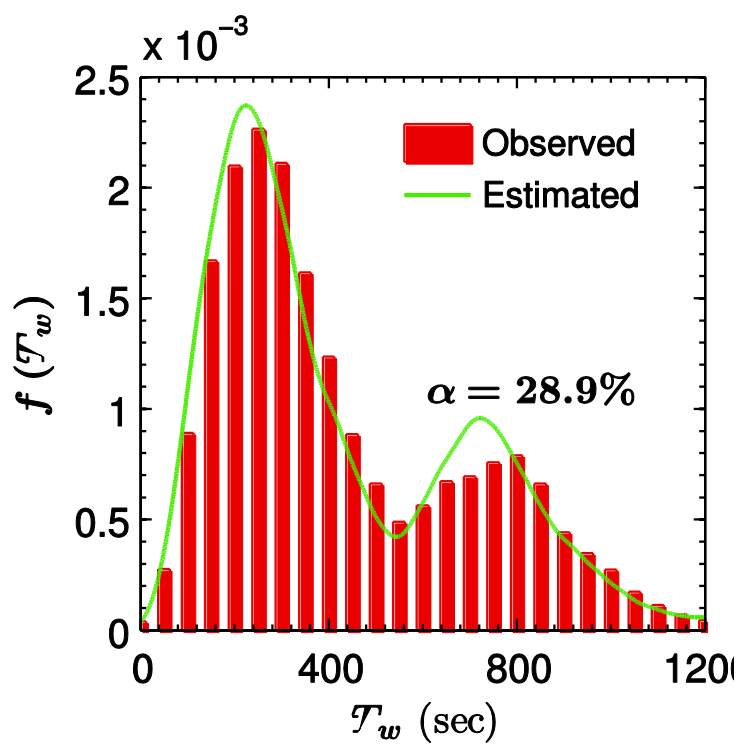

(b) p.d.f

\section{Figure 4. Observed and estimated distribution and probability density function of the waiting} time (from STN2 to STN14)

By dividing the travel time observations into slices, we can also obtain $\alpha_{B}$ for different time periods over the course of a day (the average value over five days from the dataset) by using 
the inferential model. For example, Figure 5 shows $\alpha_{B}$ from STN2 to all the stations along the metro line for different time periods. Travelling backwards is more common in the morning (between 6 am and 9 am).

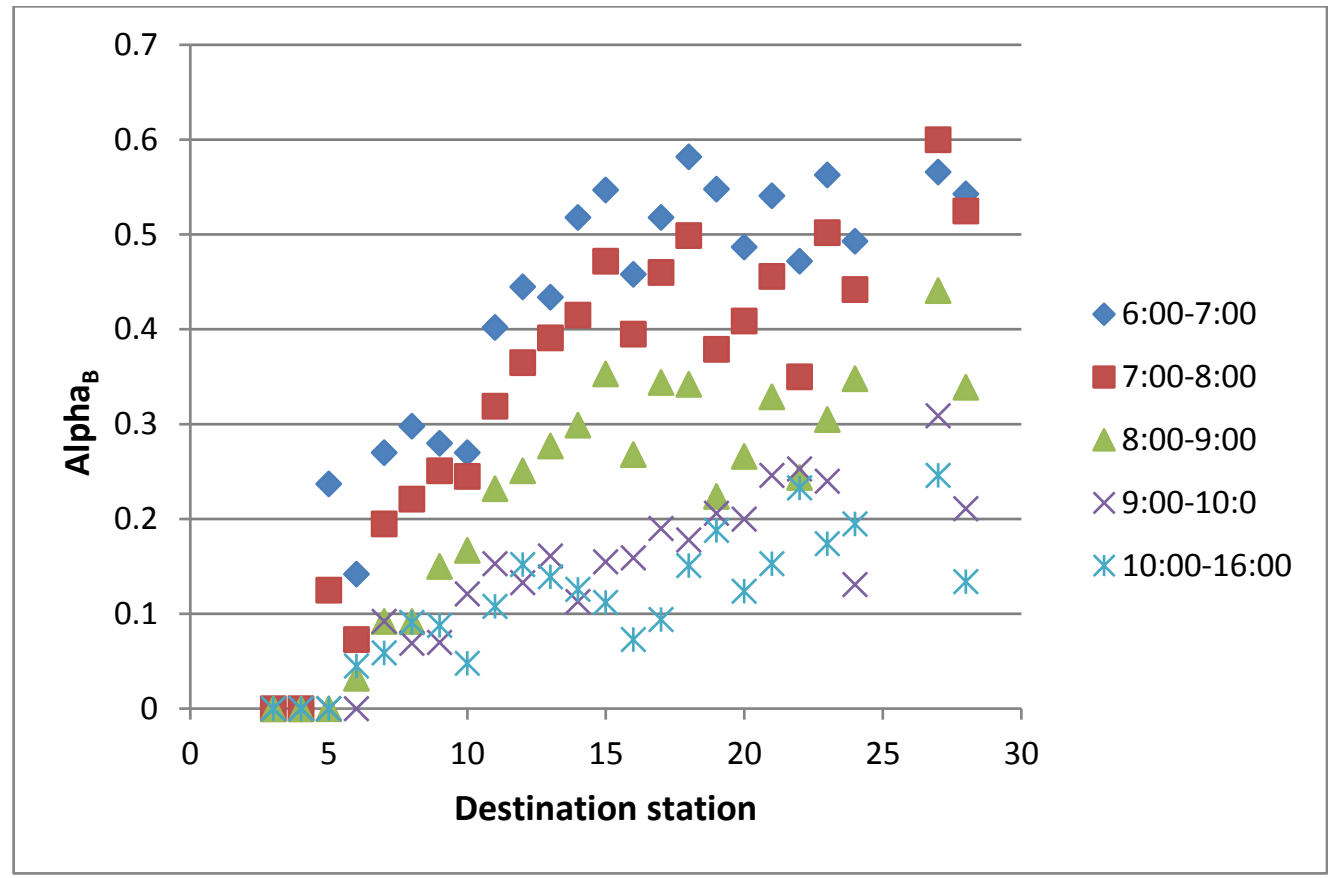

Figure 5. Spatial-temporal variation of $\alpha_{B}$ from STN2 to other stations

To finish preparing the variables necessary to estimate travel time disutility, we need to quantify train occupancy levels per section and time period, as performed in Section 3.4.

\subsection{Estimation of train loads}

After obtaining the parameters of the proposed travel time model, the temporal location of each passenger who has not transferred to another MTR line can be estimated as follows (Sun et al., 2012):

$$
L_{k}(t)=\left(T_{i j}-a_{i}-e_{j}-(j-i) \times D w\right) v+D_{k},
$$

To estimate the real train load, we route users with transfers and identify their transfer stations using the path with the shorter travel time from their origin (destination). Then, for users who travel from the studied line to other lines, their tap-in times are used to estimate their transfer times; however, for users who travel from other lines to the studied line, their tap-out times are used. Figure 8 shows the load factor (the number of passengers over the number of seats) and the density of standees for different time periods along the EW line. For example, a load factor of 3 means that all seats are occupied and that for each seated passenger, there are two 
standing passengers. An alternative way of representing occupancy or crowding conditions is through the density of standees, as depicted in Figure 9 (for a discussion on alternative crowding measures, see footnote 2 and Wardman and Whelan, 2011). Trains have 276 seats and an area of 428 square metres. To calculate the area available for standing, a seated passenger is assumed to occupy 0.4 square metres, as is recommended for longitudinal seats by TRB (2003).

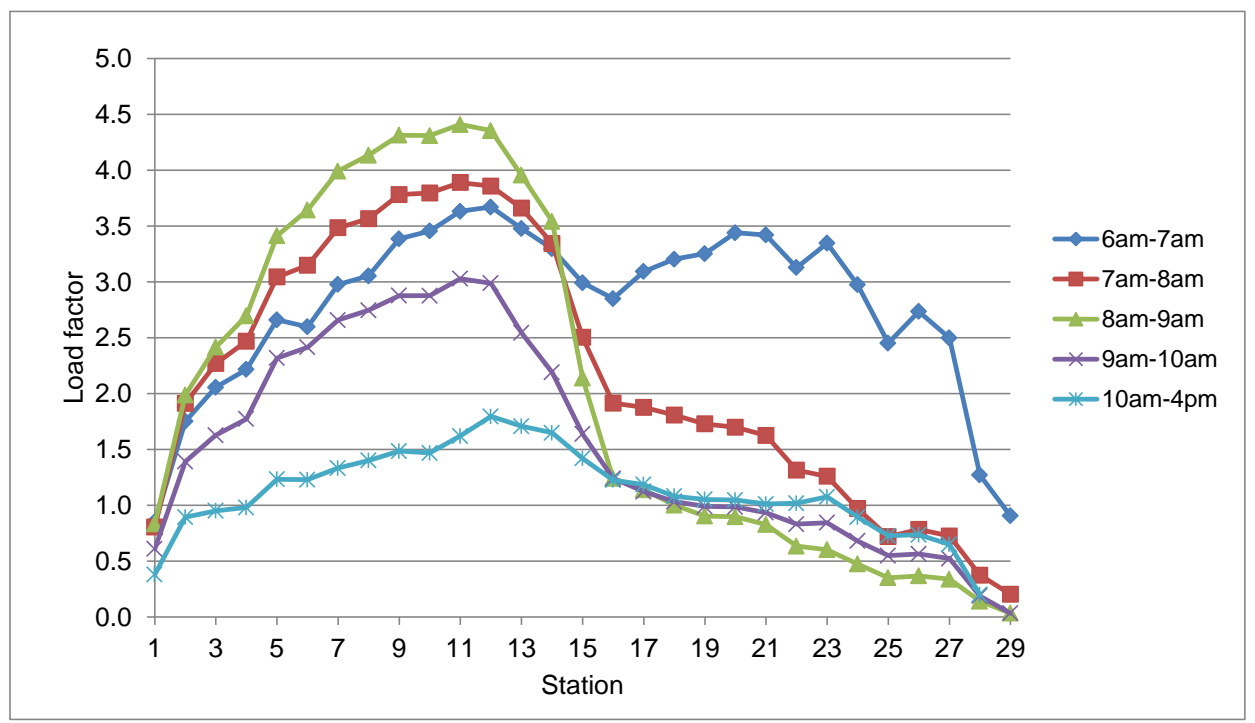

Figure 6. Average train load factor along the line for different time periods of trip departure

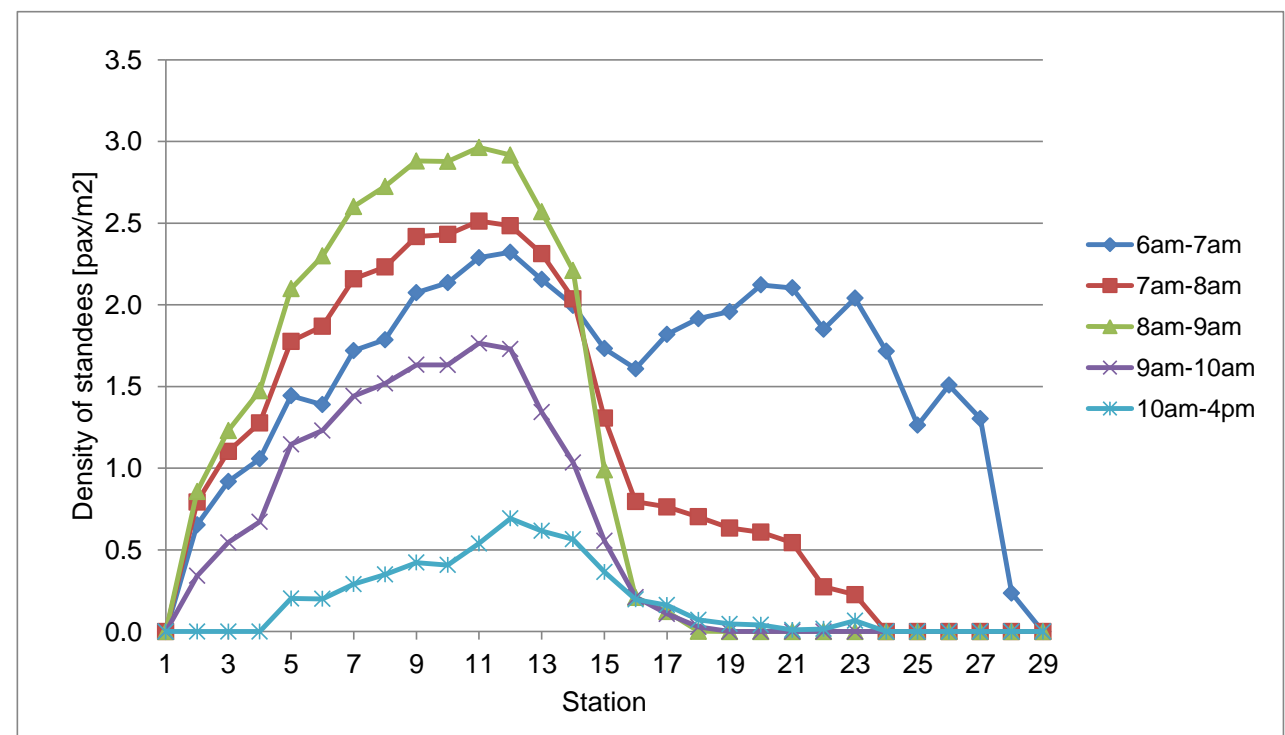

Figure 7. Average density of standees inside trains along the line for different time periods of trip departure 
Once we have estimated the proportion of passengers travelling backwards, depending on the origin and destination of each trip, the parameters associated with the utility of travel time can be estimated using discrete choice models, as shown in the next section.

\section{Estimation of discrete choice models}

In this section, we estimate discrete choice models for the choice between route alternatives (forwards or backwards) as a way of obtaining variations in the perception of time between users who prefer shorter travel times and users who are willing to trade travel time for comfort (seat availability). Logit models have usually been estimated in analyses of sitting, standing and crowding disutilities in public transport to obtain willingness-to-pay estimates for reductions in train occupancy levels. The simplest method involves the estimation of single coefficients that attempt to capture individuals' average behaviour with multinomial logit (MNL) models, as performed by Whelan and Crockett (2009), Wardman and Whelan (2011) and Tirachini et al. (2013). MNL models are simple yet powerful tools that are designed to find the average crowding and standing multipliers that attempt to represent aggregated observed behaviours, even in meta-analyses that combine several sets of parameters that are estimated with MNL models (Wardman and Whelan, 2011). An advantage of MNL models is that they can be readily introduced in public transport design problems to analyse the impact of crowding disutilities on the optimal level of variables, such as service headway, capacity and fares for buses and trains (Tirachini et al., 2014). Beyond MNL models, Tirachini et al. (2013) estimate error component (EC) logit models using crowding valuation data from Sydney, Australia, and find differences in the parameter estimates relative to MNL models; however, with EC and MNL models, the impact of crowding on the estimation of public transport demand was similar.

Let $t_{1}^{i j}$ and $t_{2}^{i j}$ be the total travel time from origin $i$ to destination $j$ for groups $\mathrm{G} 1$ (passengers travelling forwards) and G2 (passengers travelling backwards first and then forwards), respectively, such that $t_{1}^{i j}<t_{2}^{i j} \forall i, j, t^{k, k+1}$ is the travel time between consecutive stations $k$ and $k+1$ (including the dwell time at station $k+1$ ) and $t_{w}$ is the waiting time at STN1.

\subsection{Model without crowding variables}

In this section, we estimate MNL models for different time periods, without the explicit consideration of crowding variables. Origin stations are restricted to STN2, STN3 and STN4 (from STN5 and beyond, no passenger travels backwards to STN1 to switch directions). Let $U_{1}^{i j}$ and $U_{2}^{i j}$ be the utility functions of passengers from groups $\mathrm{G} 1$ and $\mathrm{G} 2$, that travel between origin $i$ and destination $j$. 


$$
\begin{aligned}
& U_{1}^{i j}=\alpha_{1}+\beta_{1} t_{1}^{i j}, \\
& U_{2}^{i j}=\beta_{2} t_{2}^{i j},
\end{aligned}
$$

where $t_{1}^{i j}=\sum_{k=i}^{j-1} t^{k, k+1}$ and $t_{2}^{i j}=\sum_{k=i}^{j-1} t^{k, k+1}+2 \sum_{k=1}^{i-1} t^{k, k+1}+t_{h}$.

Given the differences in demand composition and the load factors observed in the studied line, the models are estimated per time period, as shown in Table 2 . The choices are generated per OD pair, following the proportions of passengers travelling backwards and forwards, as estimated in the previous procedure. Without the loss of generalizability, the sample size is chosen to keep the ratio of passengers between periods constant, as the 7 am- 8 am and 8 am- 9 am periods have around three times more passengers than the 6 am-7 am and 9 am-10 am periods.

Table 2: MNL models per time period, with no crowding variables.

\begin{tabular}{|c|c|c|c|c|}
\hline Parameter & $\mathbf{6}$ am-7 am & $\mathbf{7}$ am-8 am & $\mathbf{8}$ am-9 am & 9 am-10 am \\
\hline$\alpha_{1}$ & $\begin{array}{c}-0.08 \\
(-0.45)\end{array}$ & $\begin{array}{c}0.07 \\
(0.57)\end{array}$ & $\begin{array}{c}0.76 \\
\left(4.60^{* * *}\right)\end{array}$ & $\begin{array}{c}1.61 \\
\left(4.47^{* * *}\right)\end{array}$ \\
\hline$\beta_{1}$ & $\begin{array}{c}-0.21 \\
\left(-12.55^{* * *}\right)\end{array}$ & $\begin{array}{c}-0.27 \\
\left(-24.28^{* * *}\right)\end{array}$ & $\begin{array}{c}-0.27 \\
\left(-21.41^{* * *}\right)\end{array}$ & $\begin{array}{c}-0.23 \\
\left(-7.87^{* * *}\right)\end{array}$ \\
\hline$\beta_{2}$ & $\begin{array}{c}-0.18 \\
\beta_{1} / \beta_{2}\end{array}$ & $\begin{array}{c}-0.23 \\
\left(-10.77^{* * *}\right)\end{array}$ & $\begin{array}{c}-0.22 \\
\left(-17.36^{* * *}\right)\end{array}$ & $\begin{array}{c}-0.19 \\
\left(-6.18^{* * *}\right)\end{array}$ \\
\hline $\begin{array}{c}1.18 \\
\text { Sample size and }\end{array}$ & & 1.20 & 1.23 & 1.24 \\
\hline tests & & & & \\
\hline Observations & 2729 & 8077 & 8725 & 2795 \\
\hline LL (ASC) & -1754.7 & -4676.0 & -4050.8 & -898.7 \\
\hline LL ( $\beta$ ) & -1587.8 & -4125.3 & -3613.4 & -825.9 \\
\hline Rho-sq & 0.095 & 0.118 & 0.108 & 0.081 \\
\hline
\end{tabular}


Notes: Models estimated with NLogit5; z-test in brackets

$* * *$ : Significance at the $1 \%$ level.

For all time periods, we observe that the disutility of travel time is higher for passengers who travel forwards (mostly standing) than for those that travel backwards first (mostly seated), i.e., $\left|\beta_{1}\right|>\left|\beta_{2}\right|$. Given the internal movements inside trains and the demand randomness during the one-hour periods selected for model segmentation, it is not possible to know if passengers in G1 are always standing nor if passengers in G2 are always sitting. However, the difference between $\beta_{1}$ and $\beta_{2}$ can be regarded as a good proxy to assess differences in the perceptions of travel time when sitting and standing. The bottom line is that a gain in comfort due to the expectation of occupying a seat when travelling encourages some users to choose longer travel times.

We define the standing multiplier as $S M \equiv \beta_{1} / \beta_{2}$, as presented in Table 2 for all time periods. We find that the disutility of time for passengers who travel forwards is between 18 and 24 per cent greater than that of passengers who travel backwards, which indicates the disutility of standing relative to sitting, as estimated with "observed" data. The 18-24 per cent premium for travelling forwards seems reasonable given that, in the conditions modelled, it is possible that not everyone stands going forwards, some stand on the backward route and idle time exists on the backward journey, which might be more highly valued than normal in-vehicle time.

When compared with the previous literature, we find that the SM in Table 2 is lower than the values obtained in stated choice studies in Britain but larger than the values obtained in France. A standing multiplier of 1.4 for London can be calculated with the results of Whelan and Crocket (2009), whereas a standing multiplier between 1.54 and 1.58 for commuting and leisure can be derived from the meta-analysis of British studies of Wardman and Whelan (2011). On the other hand, the ratio between standing and sitting values of time was found to be approximately 1.1 on the metro, buses, trams, and trains in the Paris region (Kroes et al., 2014). Thus, the accumulated evidence suggests that the disutility of standing over sitting can be estimated with either stated preference or revealed preference data; however, its value depends on local conditions and possibly on the modelling approach, something that requires further research.

Beyond the average values of SM per time period, the models reported in Table 2 cannot relate the SM to occupancy levels or crowding conditions because the load factors or density of standing passengers are not attributes in utility functions (11) and (12). The relationship between occupancy levels and the disutility of standing is analysed next. 


\subsection{Model including crowding variables}

We present estimation results for MNL models in which travel time interacts with crowding variables, defined as either the load factor, $L$, or the density of standing passengers, $D$. In principle, we would expect both groups G1 (likely standing) and G2 (likely sitting) to be affected by crowding levels. However, when estimating models with interaction terms of the time* crowding type for both $\mathrm{G} 1$ and $\mathrm{G} 2$, the crowding parameter for both sitting and standing are not statistically significant, possibly due to the correlation between time variables $t_{1}^{i j}$ and $t_{2}^{i j}$ . Therefore, we include crowding variables only for G1 (likely standing), whereas the time disutility of G2 (likely sitting) is left constant, regardless of the crowding levels. The implication of this assumption is evident in the results presented in Table 3.

In our modelling framework, we explicitly account for the different occupancy levels section by section along the journey, which is more realistic than the usual assumption of stated choice experiments that present a constant crowding level throughout the journey. We estimate alternative models, taking into account the density of standing passengers and the load factor as crowding attributes, as shown in Figures 8 and 9. Let $D^{k, k+1}$ and $L^{k, k+1}$ be the density of standing passengers and the load factor between stations $k$ and $k+1$, respectively. We have two alternative choice models:

$$
\begin{aligned}
& \text { D-model } \quad U_{1}^{i j}=\alpha_{1}+\beta_{1} t_{1}^{i j}+\beta_{D} t_{D 1}^{i j}, \quad U_{2}^{i j}=\beta_{2} t_{2}^{i j} \\
& \text { L-model } \quad U_{1}^{i j}=\alpha_{1}+\beta_{1} t_{1}^{i j}+\beta_{L} t_{L 1}^{i j}, \quad U_{2}^{i j}=\beta_{2} t_{2}^{i j}
\end{aligned}
$$

where $t_{D 1}^{i j}=\sum_{k=i}^{j-1} t^{k, k+1} \cdot D^{k, k+1}$ and $t_{L 1}^{i j}=\sum_{k=i}^{j-1} t^{k, k+1} \cdot L^{k, k+1}, t_{1}^{i j}$ and $t_{2}^{i j}$ defined as described above. Models (13) and (14) could not be estimated for the 6 am-7 am and 9 am-10 am periods, possibly because of the lower spatial variation in these periods' crowding levels compared with the $7 \mathrm{am}-8$ am and $8 \mathrm{am}-9$ am periods, as shown in Figure 8. The estimation results are presented in Table 3.

Table 3: MNL models per time period, with crowding variables.

\begin{tabular}{|c|c|c|c|c|}
\hline Parameter & $\mathbf{7}$ am-8 am $\boldsymbol{D}$ & $\mathbf{7}$ am-8 am $\mathbf{8}$ am-9 am $\boldsymbol{D}$ & $\mathbf{8}$ am-9 am $\mathbf{~}$ \\
\hline$\alpha_{1}$ & 0.38 & 0.30 & 1.44 & 1.39 \\
& $\left(2.82^{* * *}\right)$ & $\left(-2.24^{* *}\right)$ & $\left(7.42^{* * *}\right)$ & $\left(7.26^{* * *}\right)$ \\
\hline$\beta_{1}$ & -0.22 & -0.18 & -0.23 & -0.20 \\
\hline
\end{tabular}




\begin{tabular}{|c|c|c|c|c|}
\hline & $(-18.59 * *)$ & $\left(-13.43^{* * *}\right)$ & $\left(-17.86^{* * *}\right)$ & $\left(-14.38^{* * *}\right)$ \\
\hline$\beta_{2}$ & -0.24 & -0.24 & -0.24 & -0.23 \\
\hline$\beta_{D 1}$ & -0.049 & & $\left(-18.54^{* * *}\right)$ & $\left(-18.47^{* * *}\right)$ \\
\hline$\beta_{L 1}$ & $\left(-11.44^{* * *}\right)$ & & -0.046 & \\
& & -0.041 & & -0.037 \\
\hline Observations & 8077 & $\left.80773^{* * *}\right)$ & 8725 & 8725 \\
\hline LL (ASC) & -4676.0 & -4676.0 & -4050.9 & -4050.9 \\
\hline LL ( $\beta$ ) & -4056.4 & -4057.9 & -3538.0 & -3540.1 \\
\hline Rho-sq & 0.133 & 0.132 & 0.127 & 0.126 \\
\hline
\end{tabular}

Notes: Models estimated with NLogit5; z-test in brackets.

$* * *, * *$ : Significance at the $1 \%$ and $5 \%$ levels, respectively.

Parameters $\beta_{D 1}$ and $\beta_{L 1}$ are statistically significant at the $1 \%$ level, suggesting that train occupancy, specified either as the load factor or as the density of standing passengers per section, is significant in explaining users' choices regarding travelling forwards or backwards to secure a seat. Negative values for $\beta_{D 1}$ and $\beta_{L 1}$ indicate that the disutility of standing relative to sitting increases as trains become more crowded.

With this model, we are able to obtain standing multipliers (SMs) as a function of the load factor, $L$, or the density of standing passengers, $D$ :

$$
S M_{L}=\frac{\beta_{1}+\beta_{L 1} L}{\beta_{2}} \quad S M_{D}=\frac{\beta_{1}+\beta_{D 1} D}{\beta_{2}}
$$

These standing multipliers are defined in the same way as the crowding multipliers in the crowding valuation literature. Figures 10 and 11 show $S M_{D}$ and $S M_{L}$ for the studied MRT line during the 7 am-8 am and 8 am-9 am periods. In Figure 10, $S M_{D}$ is shown together with the London and South England curves elaborated with the results from Whelan and Crockett (2009), which is the only previous study in the literature that specifies crowding multipliers for sitting and standing as a function of the density of standing passengers. 
In Singapore's case, the standing multiplier $S M_{D}$ is remarkably similar for both time periods and grows to 1.55 when the density of standing passengers is 3 passengers per square metre, the maximum (average) density estimated (Figure 9). That is, with 3 passengers standing per square metre, the disutility of travel time standing is estimated to be 55 per cent greater than the disutility of travel time sitting, assuming that users in G1 stand and users in G2 sit all the time. Singapore's curves are fully obtained with observed preferences rather than with stated choice data. On the other hand, the model of Whelan and Crockett (2009) allows the estimation of separate crowding multipliers for sitting $M_{2}$ and standing $M_{1}$; therefore, the standing multiplier is calculated as $M_{1} / M_{2}$ (curve "London stand/sit" in Figure 10). Interestingly, even though both $M_{1}$ and $M_{2}$ grow with $D$, the ratio between them is almost constant, which means that the disutility of standing relative to that of sitting remains constant, irrespective of crowding levels - something that cannot hold in Singapore's model because, by construction of the model, $S M$ increases if trains are more crowded.

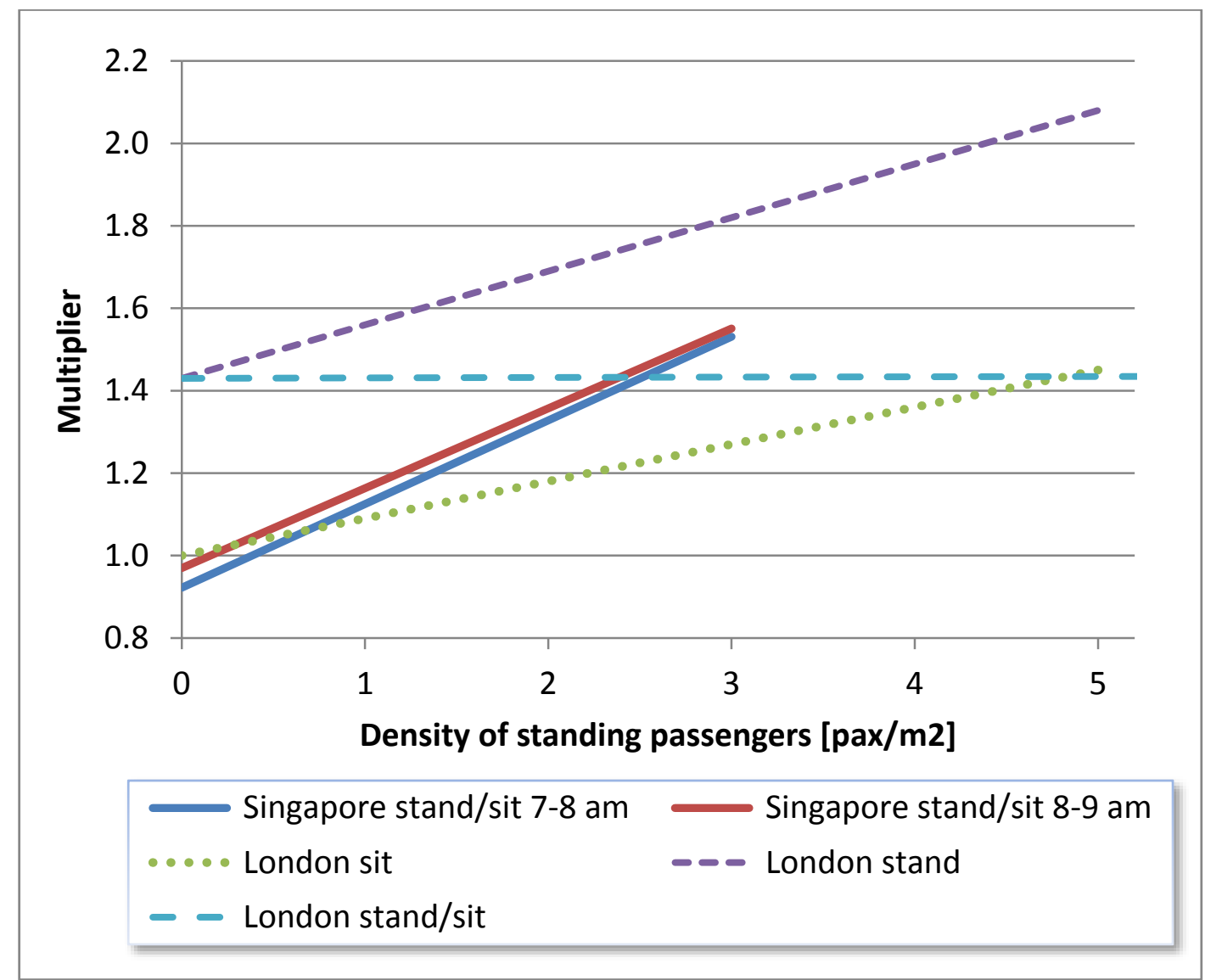

Figure 10: Singapore standing multiplier as a function of density of standees, and a comparison with the London results of Whelan and Crockett (2009) 
Figure 11 shows $S M_{L}$ and compares it to the results of the meta-analysis of British studies on crowding multipliers as a function of the load factor by Wardman and Whelan $(2011)^{5}$. Using MNL models, they find that standing $M_{1}$ and sitting $M_{2}$ multipliers are higher for leisure than for commuting ( $\mathrm{MNL}$ ); however, the ratios $M_{1} / M_{2}$ (standing multiplier) are similar for both purposes, between 1.54 and 1.58 for commuting and leisure when the load factor is between 1 and 2 . That is, on average, standing is valued a little bit over 50 per cent more than sitting, which is similar to the Singapore results only in terms of the highest occupancy rates obtained (3 standing passengers per square metre, equivalent to a load factor of 4.5 in Figures 8 and 9). However, with load factor models, comparing our Singapore results with those of other studies is less straightforward because behind the load factor, there are different configurations of sitting and standing that are specific to trains in Singapore and Britain. The higher disutilities obtained from the meta-analysis of British crowding valuation studies are likely influenced by having more seats in the average British carriage compared with the average carriage in Singapore's metro; therefore, a load factor of 2 is already crush capacity, whereas a load factor of 2 is reached, on average, with approximately 0.8 standing passengers per square metre in Singapore. Therefore, even though for sitting passengers the load factor is a relevant measure of occupancy, when there are standing passengers, the density of standees should be used for the analysis of crowding externalities, as it easies the comparability across different studies.

\footnotetext{
${ }^{5}$ Wardman and Whelan (2011) estimate multipliers for commuting and leisure trips. Only commuting is shown in Figure 11.
} 


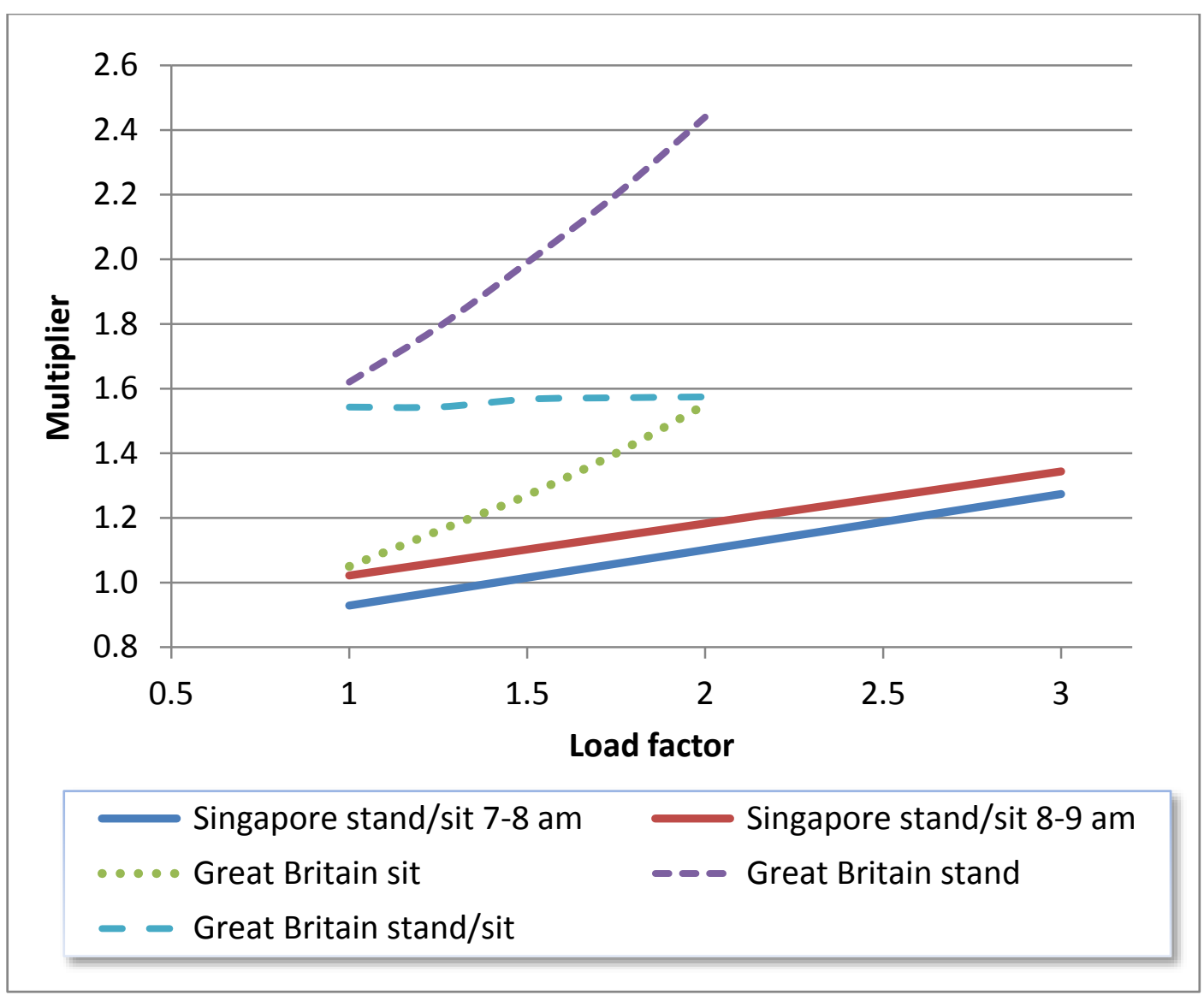

Figure 11: Singapore standing multiplier as a function of the load factor and a comparison with the Great Britain results of Wardman and Whelan (2011)

\section{Policy implications}

Because the present results on the perception of crowding are based on observed trips in public transport smart card records, it is only natural to use the same data to quantify the relevance of including perception of crowding in policy analysis. In particular, we assess the underestimation of the travel time disutility when the density of standing passengers is ignored as a crowding externality. With our framework, this assessment is performed with parameters obtained by modelling observed choices and actual train occupancy levels section by section. In the absence of an adequate estimate of the value of travel time savings in the given context, we restrict such quantification by applying the parameters resulting from Model A (sitting vs. standing without an interaction with the standing density) and Model B (with a standing density interaction) to the estimated trainload, as described in Section 3.4. For each time interval and station-to-station segment, we compute the travel time-related disutility depending on seat availability and the density of standing passengers as follows: 
If the train load between station $k$ and $k+1$ is lower than the seating capacity ( 276 seats per train), then

$$
\text { Model A and B: } \quad u^{k, k+1}=\beta_{1} t_{1}^{k, k+1} \cdot p_{\text {seat }}^{k, k+1}
$$

If the train load between station $k$ and $k+1$ is larger than the seating capacity, then

$$
\begin{array}{ll}
\text { Model A: } & u^{k, k+1}=\left(\beta_{1} \cdot p_{\text {seat }}^{k, k+1}+\beta_{2} \cdot p_{s t}^{k, k+1}\right) \cdot t^{k, k+1} \\
\text { Model B: } & u^{k, k+1}=\left(\beta_{1, B} \cdot p_{\text {seat }}^{k, k+1}+\left(\beta_{2}+\beta_{D} \cdot D^{k, k+1}\right) \cdot p_{s t}^{k, k+1}\right) \cdot t^{k, k+1}
\end{array}
$$

where $p_{\text {seat }}^{k, k+1}$ accounts for the number of seated passengers, $p_{s t}^{k, k+1}$ refers to the number of standing passengers and $D^{k, k+1}$ refers to the density of standing passengers $\left[\mathrm{pax} / \mathrm{m}^{2}\right.$ ]. Based on this calculation, we can then define the relative difference when accounting for crowding, measured in terms of standing passenger density, using an approach that only distinguishes between seating and standing when travelling as follows:

$$
\text { Model A and B: } \quad d_{r}^{k, k+1}=\frac{u_{B}^{k, k+1}-u_{A}^{k, k+1}}{u_{A}^{k, k+1}}
$$

Because Model B could only be successfully estimated for the time periods between 7 am and 8 am and between 8 am and $9 \mathrm{am}$, the relative difference in the perceived travel time disutility when accounting for the crowding of standees as obtained when applying Model $A$ and $B$, is restricted to the 7 am-9 am period, as depicted in Figure 12. 


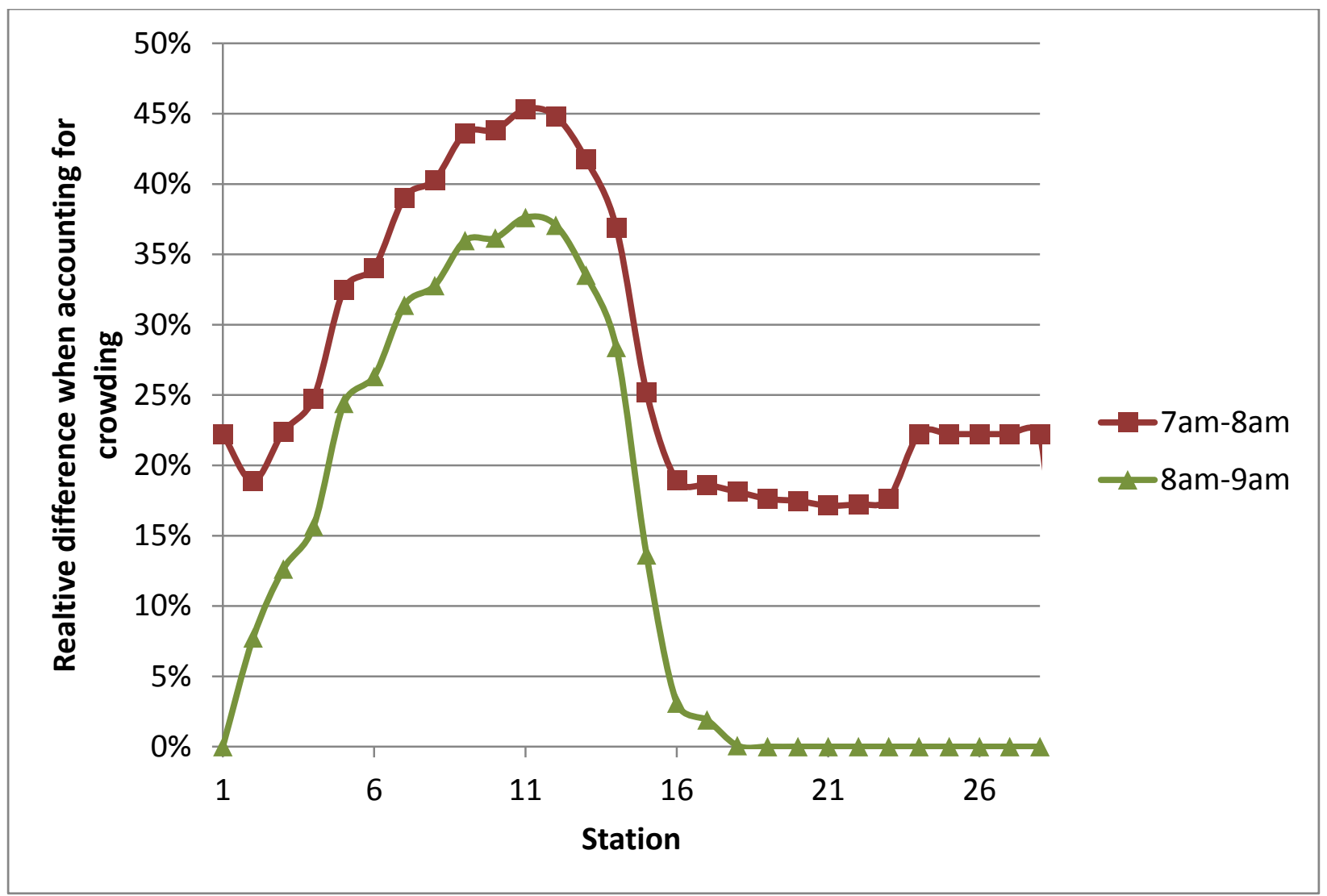

Figure 12: Relative difference in perceived travel time disutility when accounting for the crowding of standing passengers.

Not accounting for the crowding of standing passengers will underestimate travel time disutility, particularly for segments with high demand (between STN1 and STN15). While the bias amounts to $45 \%$ for the most frequently travelled segments between 7 am and 8 am, the passenger-weighted average for both time periods is $28 \%$. The values are generally higher during the earlier time period because parameter $\beta_{1}$, which multiplies travel time while seated, is considerably lower during the earlier time period in Model A than in Model B. The bias of ignoring crowding in our framework can be substantial, even if the standing multiplier found in Singapore's EW line is relatively low compared with the values obtained in other contexts, for instance, in the United Kingdom.

The main conclusion of Figure 12 is that neglecting passenger crowding effects in policy evaluations will lead to systematic underinvestment in public transport capacity upgrades to serve peak demand; by ignoring crowding, users' costs are underestimated by a non-negligible margin. Depending on the situation and type of transport system, the capacity can be enlarged by providing higher capacity vehicles or more frequent services. For a metro system, the former case is rarely an option, as train and platform lengths are usually aligned. On the other hand, 
increasing train frequency not only reduces crowding but also leads to shorter average waiting times - in turn, improving the perceived service quality (Mohring, 1972; Jara-Díaz and Gschwender, 2003). However, increasing capacity usually results in additional working hours for drivers, the need for additional vehicles, and higher energy and maintenance costs; it might even require an upgrade of the signalling system. Tirachini et al. (2014) have analysed the trade-off between users' benefits and operators' costs, which determines optimal public transport capacity levels when crowding is relevant, for urban buses.

Furthermore, ignoring the disutility of crowding will lead to an underestimation of the benefits resulting from peak-spreading strategies, such as time-differentiated pricing or the provision of (real-time) information about the crowding levels of trains. The relationship between crowding externalities and peak-spreading is particularly relevant as more and more cities are embracing such strategies: Melbourne (Currie 2010) and Singapore (Chow 2013) have introduced free travel for early morning trips, while the Washington Metropolitan Area Transit Authority introduced higher fares during peak hours in 2007 (Gwee and Currie 2013). At the same time, providing information about expected crowding levels on individual services is becoming standard in places that have already introduced systems that automate vehicle positions and passenger counting.

\section{Conclusions}

In this paper, by analysing the observed behaviour of commuters on a metro line in Singapore, we estimate taste parameters in an attempt to model the election between a longer travel time sitting versus a shorter travel time standing. travel time sitting and standing. Although the study of sitting, standing and crowding valuations has gained momentum in the literature on stated choice models (Douglas and Karpouzis, 2005; Whelan and Crockett, 2009; Hensher et al., 2011; Wardman and Whelan, 2011; Fröhlich et al., 2012; Tirachini et al., 2013; Batarce et al., 2015), inferring the relative impact of standing and crowding levels on travel behaviour has been difficult because of the lack of tools capable of monitoring individuals' actual choices. We take advantage of the emerging public transport smart card data as a proxy to estimate the differences in travel time valuations between passengers who are willing to experience longer travel times if seated and passengers who prefer shorter travel times, even if they have to stand for at least part of their trip. In other words, unlike the previous literature, our study is based on modelling the observed choices of a set of travellers who prefer to first travel backwards (in the opposite direction of their destinations) in an attempt to secure a seat in the forward direction during the morning rush. 
We estimate the disutility of travel time standing to be, on average, between $18 \%$ and $24 \%$ greater than the disutility of travel time sitting for morning long-distance passengers on the EW metro line in Singapore. By including crowding measures, i.e., the trainload factor or the density of standing passengers, we find that the standing multiplier (the ratio of the parameter of time standing to the parameter of time sitting) increases from 1, when there are no standing passengers, to 1.55 , when there are 3 standing passengers per square metre. These values are compared with those of previous studies from Great Britain and France. One limitation of our study is that, because of the internal movement inside trains and demand randomness, it is impossible to know with certainty whether the passengers who decide to travel backwards first and then forwards are seated and whether the passengers who decide to travel forwards only stand. However, the difference between the estimated travel time parameters of both groups is regarded as a good proxy for assessing differences in the perceptions of travel time when sitting and standing, given that a gain in comfort (the expectation of securing a seat) is what drives some passengers to choose longer travel times.

Applying our model to the current load profile of the EW line quantifies the underestimation of users' travel costs that arises when passenger crowding is ignored in analysing users' public transport choices. Such an exercise can be used to inform policymakers about the optimal level of transport supply with regard to vehicle size and frequency and the value of peak-spreading strategies that reduce crowding in peak periods.

Further research is needed to understand the differences reported in the crowding valuation literature, specifically to what extent the differences observed in the literature can be explained by local circumstances or by the peculiarities of each modelling approach that analysts use. Therefore, particular emphasis should be placed on quantifying the impact of various factors that describe such circumstances on the valuation of crowding in public transport. Such factors can range from socio-demographic attributes, the trip purpose, the trip distance, the passenger's inclination and ability to perform activities when travelling (e.g., reading and using portable devices), personal attitudes, the vehicle's layout and design and cultural values and context. For example, people who really dislike standing, as well as commuting time in general, will likely choose locations that are closer to work if they have the chance, or they will choose jobs that allow them flexible arrival and departure times (or to work at home), thus avoiding peak commuting times. This sorting behaviour, if it exists, is embedded in our results, introducing a bias that has not been modelled due to the lack of information on this and other types of personal preferences. A wide range of attributes influences people's valuations of sitting, standing and crowding; therefore, many more research efforts are necessary to achieve a more comprehensive understanding of the effects of crowding externalities on user behaviours, wellbeing and choices. 


\section{Acknowledgements}

This research has been supported by Fondecyt Chile Grant 11130227, "Social effects and Quality of Service Valuation in Public Transport Services", the Complex Engineering Systems Institute, Chile (Grants ICM P-05-004-F, CONICYT FBO16) and the National Science Foundation of Singapore. We thank Kay Axhausen and two anonymous reviewers for their valuable comments, which have significantly improved this article. Any remaining errors are the authors' responsibility alone.

\section{References}

Basu, D. and J. D. Hunt, 2012. Valuing of attributes influencing the attractiveness of suburban train service in Mumbai city: A stated preference approach. Transportation Research Part A: Policy and Practice 46(9): 1465-1476.

Batarce, M., J. C. Muñoz, J. d. D. Ortúzar, S. Raveau, C. Mojica and R. A. Rios, 2015. Valuing crowding in public transport systems using mixed stated/revealed preferences data: the case of Santiago. 94th TRB Annual Meeting, Washington D.C.

Bates, J., J. Polak, P. Jones and A. Cook, 2001. The valuation of reliability for personal travel. Transportation Research Part E 37(2-3): 191-229.

Börjesson, M., J. Eliasson and J. P. Franklin, 2012. Valuations of travel time variability in scheduling versus mean-variance models. Transportation Research Part B 46(7): 855-873.

Chakirov, A. and A. Erath, 2011. Use of public transport smart card fare payment data for travel behaviour analysis in Singapore. 16th International Conference of Hong Kong Society for Transportation Studies. Hong Kong.

Dempster, A. P., N. M. Laird and D. B. Rubin, 1977. Maximum likelihood from incomplete data via the EM algorithm. Journal of the Royal Statistical Society. Series B (Methodological): 1-38.

Douglas, N. and G. Karpouzis, 2005. Estimating the cost to passengers of station crowding. 28th Australasian Transport Research Forum (ATRF), Sydney, September 2005.

Douglas, N. and G. Karpouzis, 2006. Estimating the passenger cost of train overcrowding. 29th Australasian Transport Research Forum, Gold Coast, September 2006.

Fröhlich, P., K. W. Axhausen, M. Vrtic, C. Weis and A. Erath, 2012. SP-Befragung 2010 zum Verkehrsverhalten im Personenverkehr, Swiss Federal Office for Spatial Development, Bern.

Hasan, S., C. M. Schneider, S. V. Ukkusuri and M. C. González, 2012. Spatiotemporal Patterns of Urban Human Mobility. Journal of Statistical Physics 1: 245.

Hensher, D. A., J. M. Rose and A. Collins, 2011. Identifying commuter preferences for existing modes and a proposed Metro in Sydney, Australia with special reference to crowding. Public Transport 3(2): 109147. 
Jara-Díaz, S. R. and A. Gschwender, 2003. Towards a general microeconomic model for the operation of public transport. Transport Reviews 23(4): 453 - 469.

Kim, J.-K., B. Lee and S. Oh, 2009. Passenger choice models for analysis of impacts of real-time bus information on crowdedness. Transportation Research Record 2112: 119-126.

Kroes, E., M. Kouwenhoven, L. Debrincat and N. Pauget, 2014. Value of Crowding on Public Transport in Île-de-France, France. Transportation Research Record 2417: 37-45.

Li, Z. and D. A. Hensher, 2011. Crowding and public transport: A review of willingness to pay evidence and its relevance in project appraisal. Transport Policy 18(6): 880-887.

Lin, T. and N. H. M. Wilson, 1992. Dwell time relationships for light rail systems Transportation Research Record 1361: 287-295.

LT Marketing, 1988. Research into Crowding Penalties for LUL and LRT.

Mohring, H., 1972. Optimization and scale economies in urban bus transportation. American Economic Review 62(4): 591-604.

Munizaga, M., A. and C. Palma, 2012. Estimation of a disaggregate multimodal public transport OriginDestination matrix from passive smartcard data from Santiago, Chile. Transportation Research Part C 24: 9-18.

OECD/ITF, 2014. Valuing Convenience in Public Transport. ITF Round Tables, No. 156, OECD Publishing. .

Pelletier, M. P., M. Trépanier and C. Morency, 2011. Smart card data use in public transit: A literature review. Transportation Research Part C 19: 557-568.

Raveau, S., J. C. Muñoz and L. de Grange, 2011. A topological route choice model for metro. Transportation Research Part A: Policy and Practice 45(2): 138-147.

Sun, L., K. W. Axhausen, D.-H. Lee and X. Huang, 2013. Understanding metropolitan patterns of daily encounters. Proceedings of the National Academy of Sciences of the United States of America 110(34): 13774-13779.

Sun, L., D.-H. Lee, A. Erath and X. Huang (2012). Using smart card data to extract passenger's spatiotemporal density and train's trajectory of MRT system. ACM SIGKDD International Workshop on Urban Computing, ACM.

Sun, L., D.-H. Lee, A. Erath and X. Huang (2012). Using smart card data to extract passenger's spatiotemporal density and train's trajectory of MRT system. Proceedings of the ACM SIGKDD International Workshop on Urban Computing, ACM.

Theler, B. and K. W. Axhausen, 2013. When is a bus full? A study of perception, Arbeitsberichte Verkehrs- und Raumplanung, 855, IVT, ETH Zurich, Zurich.

Timmins, C. and J. Murdock, 2007. A revealed preference approach to the measurement of congestion in travel cost models. Journal of Environmental Economics and Management 53(2): 230-249.

Tirachini, A., D. A. Hensher and J. M. Rose, 2013. Crowding in public transport systems: effects on users, operation and implications for the estimation of demand. Transportation Research Part A 53: 36-52. 
Tirachini, A., D. A. Hensher and J. M. Rose, 2014. Multimodal pricing and optimal design of urban public transport: the interplay between traffic congestion and bus crowding. Transportation Research Part $B$ 61: 33-54.

TRB, 2003. Transit Capacity and Quality of Service Manual. TCRP Report 100.

Wardman, M. and G. A. Whelan, 2011. Twenty years of rail crowding valuation studies: evidence and lessons from British experience. Transport Reviews 31(3): 379-398.

Whelan, G. and J. Crockett (2009). An investigation of the willingness to pay to reduce rail overcrowding. International Conference on Choice Modelling, Harrogate, England, April. 